\title{
Corrélats de l'âge au moment du diaǵnostic de troubles du spectre autistique dans six régions canadiennes
}

\author{
H. Coo, M. Sc. (1, 2); H. Ouellette-Kuntz, M. Sc. (1, 2, 3); M. Lam, Ph. D. (1); C. T. Yu, Ph. D. (2, 4, 5); \\ D. Dewey, Ph. D. (2, 6, 7, 8); F. P. Bernier, M.D. (2, 9); A. E. Chudley, M.D. (2, 10, 11); P. E. Hennessey, B.A. (12); \\ M. M. Breitenbach, M. S. Éd. (13); A. L. Noonan, M.A. (14); M. E. Lewis, M.D. (2, 15), J. J. Holden, Ph. D. (2, 3, 16, 17)
}

Cet article a fait l'objet d'une évaluation par les pairs.

\section{Résumé}

Introduction : La détection précoce des troubles du spectre autistique (TSA) est importante, étant donné qu'une exposition rapide à des programmes d'intervention comportementale peut améliorer les résultats pour l'enfant. De plus, elle permet aux familles d'accéder rapidement à d'autres traitements et services de soutien.

Méthodologie : À l'aide d’une modélisation linéaire généralisée, nous avons examiné l'association entre les caractéristiques de l'enfant et de la famille et l'âge auquel 2180 enfants ont reçu un diagnostic de TSA entre 1997 et 2005 dans six régions du Canada.

Résultats : Un diagnostic de trouble envahissant du développement non spécifié (TED-NS) ou de syndrome d'Asperger, le fait d'habiter en milieu rural, un diagnostic posé récemment et le fait d'être né à l'étranger étaient associés à un âge avancé au moment du diagnostic. Les enfants appartenant à une minorité visible ou ayant une sœur ou un frère atteint de TSA étaient plus nombreux à recevoir un diagnostic précoce. Toutefois, considérés dans leur ensemble, ces facteurs ne suffisent pas à expliquer la variation de l'âge au moment du diagnostic.

Conclusion : S'il est encourageant de constater que l'identité ethnoculturelle, le revenu du quartier, le fait d'habiter en milieu urbain ou rural et le sexe de l'enfant ne constituaient pas des facteurs majeurs contribuant aux disparités liées à l'âge auquel les enfants reçoivent un diagnostic de TSA, il y a tout de même lieu de poursuivre les recherches afin de déterminer les facteurs qui expliquent vraiment les différences observées. Les variations régionales des effets de plusieurs facteurs laissent croire que l'agrégation des données ne constitue peut-être pas une stratégie optimale si les conclusions visent à orienter les politiques et la pratique clinique à l'échelle locale.

Mots-clés : trouble du spectre autistique, âge au moment du diagnostic, surveillance, Canada, syndrome d'Asperger, trouble autistique, trouble envahissant du développement de l'enfant

\section{Introduction}

Les Centers for Disease Control and Prevention, l'American Academy of Pediatrics et les Instituts de recherche en santé du Canada soulignent tous l'importance de reconnaître les enfants atteints de troubles du spectre autistique (TSA) à l'âge le plus précoce possible ${ }^{1-3}$. Une étude récente décrit les avantages d'un diagnostic précoce ${ }^{4}$, entres autres un accès rapide à des programmes d'intervention qui contribuent à améliorer le fonctionnement intellectuel et le comportement adaptatif ${ }^{5}$. L'exposition précoce à ces programmes augmente la probabilité que l'intervention [traduction] " modifie la trajectoire développementale anormale des personnes atteintes de TSA, ramène le développement cérébral et comportemental vers la voie normale et, dans certains cas, prévienne le syndrome complet de TSA $»^{6}$. L'idée selon laquelle "le plus tôt est le mieux » est étayée par des études indiquant qu'une intervention précoce améliore l'issue clinique pour l'enfant ${ }^{7,8}$. Au Canada, le temps requis pour diagnostiquer un TSA peut également avoir une incidence sur le financement du traitement et, par conséquent, sur l'accès à celui-ci. En

\section{Rattachement des auteurs :}

1. Department of Community Health and Epidemiology, Queen's University, Kingston (Ontario), Canada

2. Autism Spectrum Disorders-Canadian-American Research Consortium (ASD-CARC), Kingston (Ontario), Canada

3. Ongwanada Resource Centre, Kingston (Ontario), Canada

4. Department of Psychology, University of Manitoba, Winnipeg (Manitoba), Canada

5. St. Amant Research Centre, Winnipeg (Manitoba), Canada

6. Department of Pediatrics, University of Calgary, Calgary (Alberta), Canada

7. Department of Community Health Sciences, University of Calgary, Calgary (Alberta), Canada

8. Behavioural Research Unit, Alberta Children's Hospital, Calgary (Alberta), Canada

9. Department of Medical Genetics, University of Calgary, Calgary (Alberta), Canada

10. Medical Genetics Research Group, University of Manitoba, Winnipeg (Manitoba), Canada

11. Department of Pediatrics and Child Health, University of Manitoba, Winnipeg (Manitoba), Canada

12. Ministère de la Santé et des Services communautaires, St. John's (Terre-Neuve-et-Labrador), Canada

13. Ministère de l'Éducation et du Développement de la petite enfance, Charlottetown (île-du-Prince-Édouard), Canada

14. Ministère des Services communautaires, des Aînés et du Travail, Charlottetown (île-du-Prince-Édouard), Canada

15. Department of Medical Genetics, University of British Columbia, Vancouver (Colombie-Britannique), Canada

16. Department of Psychiatry, Queen's University, Kingston (Ontario), Canada

17. Department of Physiology, Queen's University, Kingston (Ontario), Canada

Correspondance : Hélène Ouellette-Kuntz, Département de santé communautaire et d'épidémiologie, Université Queen's, 191 avenue Portsmouth, Kingston (Ontario) K7M 8A6; tél. : 613-548-4417; téléc. : 613-548-8135; courriel : helene.kuntz@queensu.ca 
Colombie-Britannique, par exemple, le gouvernement accorde annuellement jusqu'à 22000 \$ aux familles qui ont un enfant atteint de TSA jusqu'à ce que ce dernier atteigne l'âge de 6 ans, âge à compter duquel le montant est réduit à 6000 \$ par année? .

La détection précoce procure d'autres avantages. En effet, les parents disent se sentir soulagés quand le diagnostic est posé, car il leur permet de mieux comprendre le comportement de leur enfant ${ }^{10}$. Le diagnostic rend également l'enfant admissible à des services et à de l'aide, en plus de donner aux parents la possibilité de demander une consultation génétique.

Même s'il est souvent possible de poser de façon fiable un diagnostic de TSA quand un enfant est âgé de 2 à 3 ans ${ }^{11,12}$, l'état de nombreux enfants n'est pas diagnostiqué avant l'âge de 4 ans ou même plus tard ${ }^{1,13,14}$. Dans un tel contexte, des campagnes nationales ont été lancées au Canada et aux États-Unis en vue de promouvoir la détection précoce des $\operatorname{TSA}^{15, *}$.

Les facteurs expliquant les variations de l'âge au moment du diagnostic de TSA n'ont pas fait l'objet d'une étude approfondie; toutefois, le lieu géographique semble jouer un rôle. Une étude récente a fait état de différences dans l'âge médian au moment du diagnostic dans quatre régions du Canada ${ }^{14}$, et une étude en population réalisée aux États-Unis a révélé des différences dans 13 sites de surveillance quant à l'âge auquel les enfants avaient reçu un diagnostic initial de $\mathrm{TSA}^{16}$. Pour d'autres facteurs, les résultats sont moins constants. Dans une étude, les garçons avaient reçu un diagnostic beaucoup plus tôt que les filles ${ }^{16}$, tandis que dans d'autres études, aucune différence n'avait été signalée entre les sexes relativement à l'âge au moment du diagnostic ${ }^{17-22}$. Le fait de vivre en milieu rural et un faible revenu familial étaient associés à un diagnostic plus tardif dans une enquête réalisée auprès de 969 personnes ayant la charge d'enfants atteints de TSA en
Pennsylvanie ${ }^{17}$, tandis qu'aucune différence importante n'avait été signalée pour ces mêmes facteurs dans une enquête en ligne à laquelle avaient participé 146 personnes s'occupant d'enfants atteints de TSA en Virginie $^{18}$. Une étude portant sur l'examen de demandes de règlement de Medicaid à Philadelphie a révélé que les enfants de race blanche atteints d'un trouble autistique recevaient un diagnostic beaucoup plus tôt que les enfants afro-américains ${ }^{22}$. À l'opposé, d'après l'enquête mentionnée précédemment auprès de 969 dispensateurs de soins en Pennsylvanie, on n'a constaté aucune différence importante entre les enfants de race blanche et ceux appartenant à une minorité ethnique quant à l'âge au moment du diagnostic ${ }^{17}$. De même, les données du Metropolitan Atlanta Developmental Disabilities Surveillance Program [programme de surveillance des troubles développementaux de la région métropolitaine d'Atlanta] n'ont indiqué aucun effet important de la race ou de l'origine ethnique sur le moment du diagnostic de TSA $^{13}$.

Comme la majorité de ces études ont été réalisées aux États-Unis, il est important de les reproduire dans d'autres régions avec des échantillons de taille suffisamment grande pour tenir compte de nombreux facteurs simultanément. Les analyses présentées dans cet article ont utilisé des données recueillies par un programme canadien de surveillance des TSA. Nous avions pour objectif d'examiner l'association entre les caractéristiques de l'enfant et de la famille et l'âge auquel des enfants de six régions du Canada avaient reçu un diagnostic initial de TSA entre 1997 et 2005. Un autre objectif consistait à déterminer s'il y avait des effets différentiels des caractéristiques examinées d'une région à l'autre et d'un sous-groupe de diagnostic à l'autre.

\section{Méthodologie}

\section{Échantillon}

L'échantillon était composé de 2180 enfants issus de six régions du Canada, pour lesquels des renseignements sont enregistrés dans la base de données épidémiologique nationale pour l'étude de l'autisme au Canada (National Epidemiologic Database for the Study of Autism in Canada NEDSAC). La NEDSAC a été créée en 2001, et la surveillance des cas de TSA a commencé en 2002 chez les enfants de moins de 18 ans au Manitoba et à l'Îledu-Prince-Édouard, et en 2003 chez les enfants de moins de 15 ans dans le sud-est de l'Ontario ${ }^{\dagger}$ et à Terre-Neuve-et-Labrador. En 2002, trois centres provinciaux de référence et d'évaluation situés à Vancouver, en Colombie-Britannique, ont entrepris un examen des dossiers afin de déceler les cas de TSA chez les enfants de moins de 15 ans. La collecte de renseignements sur des cas diagnostiqués grâce à l'examen des dossiers s'est poursuivie jusqu'en 2007, date de son arrêt en Colombie-Britannique. De 2003 à 2006, la clinique de développement de l'Alberta Children's Hospital de Calgary et diverses sources communautaires de cette ville ont également fourni à la NEDSAC des données sur les enfants de moins de 15 ans atteints de TSA. Les renseignements détaillés sur les protocoles de détermination des cas et de collecte des données dans les régions sont disponibles dans le rapport d'étape publié dans ce numéro ${ }^{23}$.

Pour pallier le fait que les dates de début et les groupes d'âge ciblés étaient différents selon les régions faisant l'objet d'une surveillance, l'échantillon a englobé les enfants nés en 1989 ou après et ayant reçu un diagnostic initial de TSA avant leur $15^{\mathrm{e}}$ anniversaire. Nous avons également restreint l'échantillon à ceux qui avaient initialement reçu un diagnostic entre 1997 et 2005 inclusivement; relativement peu d'enfants avaient obtenu un diagnostic avant 1997, et la dernière année complète de collecte de données pour la ville de Calgary était 2005. La figure 1 illustre le processus de sélection de l'échantillon.

\section{Traitement statistique}

La NEDSAC renferme des renseignements sociodémographiques et diagnostiques de base sur les enfants atteints de TSA. Nous

\footnotetext{
* http://www.autismspeaks.org/press/cdc_awareness_campaign.php [en anglais]

${ }^{+}$Comprend six comtés : Hastings, Prince Edward, Lennox et Addington, Frontenac, Leeds-Grenville et Lanark.
} 
FIGURE 1

\section{Sélection de l'échantillon}

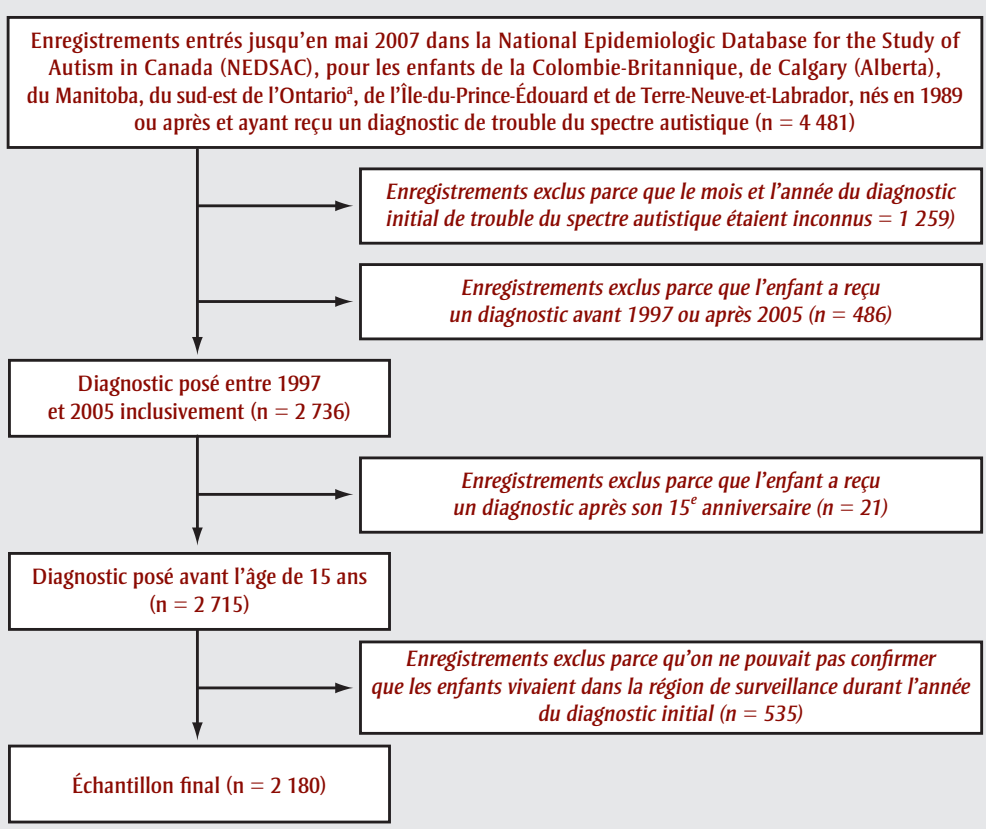

${ }^{a}$ Comprend six comtés : Hastings, Prince Edward, Lennox et Addington, Frontenac, Leeds-Grenville et Lanark.

avons examiné les caractéristiques suivantes qui pourraient être associées à l'âge au moment du diagnostic :

1) le sous-groupe de diagnostic (trouble autistique/trouble envahissant du développement non spécifié [TED-NS]/ syndrome d'Asperger/trouble du spectre autistique, cette dernière catégorie ayant été utilisée lorsqu’un diagnostic général de TSA était donné au parent ou inscrit au dossier de l'enfant);

2) l'identité ethnoculturelle, déclarée par les parents ou inscrite au dossier de l'enfant (race blanche/minorité visible/ autochtone; un enfant membre d'une minorité visible autre qu'autochtone, mais également identifié comme Autochtone entrait dans la catégorie des Autochtones);

3) le fait d'être adopté (non/oui);

4) le revenu médian des ménages du quartier, comme mesure substitutive du revenu des ménages (tertile inférieur/ moyen/supérieur, selon les renseignements du recensement canadien pour tous les ménages privés dans la région, délimitée par les trois premiers caractères du code postal du dernier lieu de résidence connu dans la région de surveillance ${ }^{24}$ );
5) le dernier lieu de résidence connu dans la région de surveillance (milieu urbain/rural, défini par le deuxième caractère du code postal; un code postal comportant un « 0 » comme deuxième caractère est classé comme rural et tous les autres codes postaux sont considérés comme urbains ${ }^{25}$ );

6) le sexe (garçon/fille);

7) l'intervalle de trois ans pour l'année du diagnostic initial (1997-1999/2000-2002 /2003-2005);

8) le lieu de naissance (Canada/autre pays); 9) le fait d'avoir une sœur ou un frère qui a reçu un diagnostic de TSA (non/oui).

La région a été traitée comme une covariable dans les modèles pour tenir compte de ses effets sur l'âge au moment du diagnostic, mais ce n'était pas une variable à l'étude. Par conséquent, aucun résultat relatif à la région ne figure dans les tableaux ou le texte. À priori, nous avons décidé de n’inclure que les principaux effets dans les modèles de régression, et nous n'avons pas testé les interactions entre les variables.

Nous avons ajusté les modèles de régression linéaire généralisée pour l'échantillon total en utilisant le système d'analyse statistique SAS version 9.1.3 (SAS Institute Inc.) et en précisant la fonction de lien logarithmique et la distribution gamma ${ }^{26}$. Afin de réduire les hypothèses concernant la distribution de la variable dépendante (âge au moment du diagnostic), nous avons utilisé la méthode de quasi-vraisemblance (ou d'équation estimante généralisée) plutôt que celle de la vraisemblance complète. Le premier modèle ne comprenait que les observations pour lesquelles des renseignements complets étaient disponibles ( "modèle de cas complet »). Quelques-unes des variables examinées comportaient beaucoup de valeurs manquantes (voir le tableau 1; les valeurs manquantes variaient d'aucune pour le sous-groupe de diagnostic, le sexe et l'année du diagnostic initial à 22,9\% pour l'identité ethnoculturelle). Même si les méthodes d'imputation ont leurs limites, les ouvrages de statistique actuels recommandent que les valeurs manquantes soient imputées plutôt que de limiter les analyses aux cas comportant des données complètes ${ }^{27}$. Nous avons utilisé la méthode de Monte Carlo par chaîne de Markov $^{28}$ afin de générer 10 imputations pour chaque point de données manquant. Les valeurs imputées n’ont pas été arrondies $^{29}$. Les modèles de régression linéaire généralisée ont par la suite été ajustés aux données imputées, et les estimations finales des paramètres ont été résumées par la méthode SAS PROC MIANALYZE (" modèles imputés »). Les valeurs $R^{2}$, qui, dans une régression linéaire, servent à indiquer la proportion de la variation de la variable dépendante expliquée par les variables indépendantes, ont été calculées à l'aide de la formule suivante : 1 - (somme brute des carrés des résidus)/(somme totale des carrés) ${ }^{30}$.

Nous avons ensuite ajusté des modèles de régression distincts pour les données de la Colombie-Britannique et du Manitoba en vue de déterminer si les effets des variables indépendantes variaient entre ces régions. Les quatre autres régions n’ont pas été incluses dans cette analyse parce que les chiffres figurant dans les cellules pour certaines variables étaient trop faibles pour produire des estimations de paramètres valables. Nous avons également ajusté des modèles distincts pour les trois sous-groupes de diagnostic (le " trouble du spectre autistique » a été exclu de cette analyse) 
TABLEAU 1

Distribution de fréquence et âges moyens et médians au moment du diagnostic pour les variables indépendantes incluses dans les analyses de régression multiple en vue d'examiner les associations avec l'âge au moment du diagnostic initial de trouble du spectre autistique (TSA)

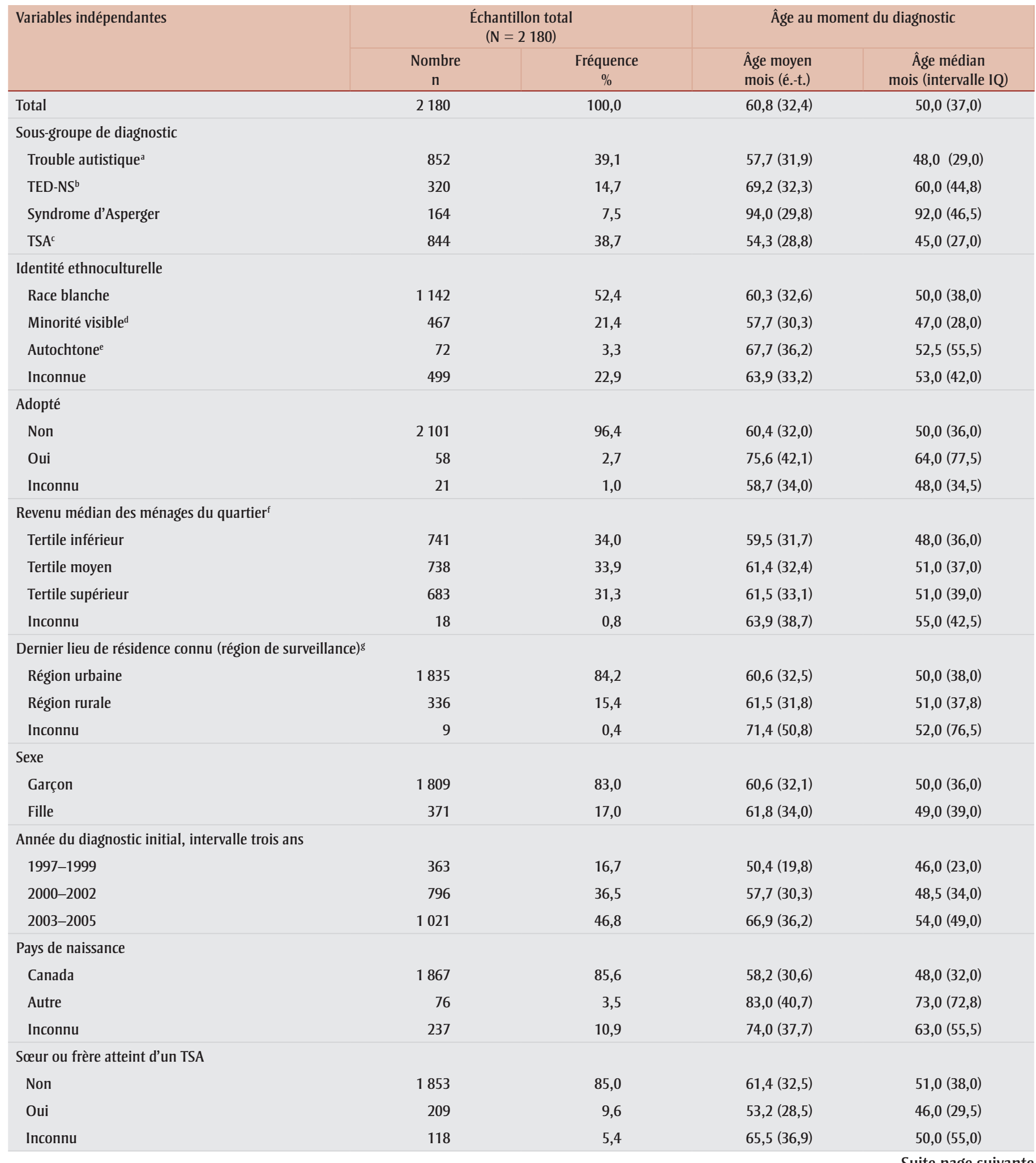




\section{TABLEAU 1 (Suite)}

Distribution de fréquence et âges moyens et médians au moment du diagnostic pour les variables indépendantes incluses dans les analyses de régression multiple en vue d'examiner les associations avec l'âge au moment du diaǵnostic initial de trouble du spectre autistique (TSA)

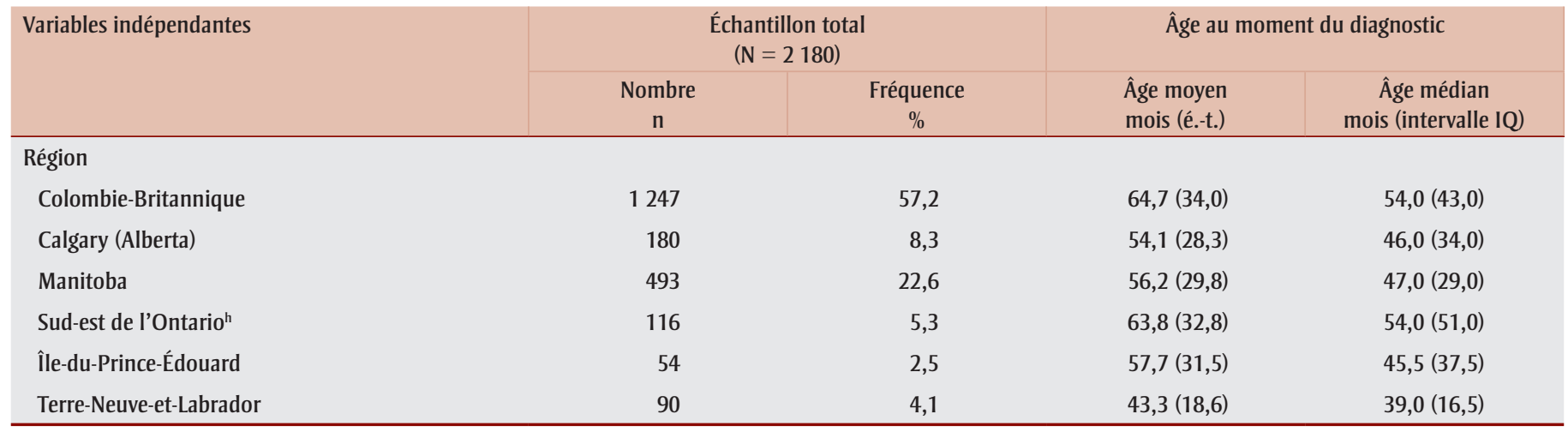

Abréviations : é.-t., écart-type; IQ, interquartile; NEDSAC, National Epidemiologic Database for the Study of Autism in Canada; TED-NS, trouble envahissant du développement non spécifié; TSA, trouble du spectre autistique.

${ }^{a}$ Comprend les enfants ayant reçu un diagnostic d'autisme de la petite enfance et d'autisme infantile.

${ }^{b}$ Comprend les enfants ayant reçu un diagnostic de trouble envahissant du développement apparu dans l'enfance, d'autisme atypique et d'autres troubles envahissants du développement.

c Diagnostic général de trouble du spectre autistique fourni aux parents ou inscrits au dossier de l'enfant.

${ }^{\mathrm{d}}$ Exclut l'identité autochtone.

e Premières Nations/Amérindiens, Inuits ou Métis. Au Manitoba, la population dans les réserves n'est pas incluse dans la NEDSAC. Les réserves sont de compétence fédérale et ne sont pas desservies par l'organisme manitobain qui détermine les cas pour la NEDSAC.

${ }^{f}$ Fondé sur le revenu médian des ménages pour tous les ménages privés dans la région délimitée par les trois premiers caractères du code postal ${ }^{24}$.

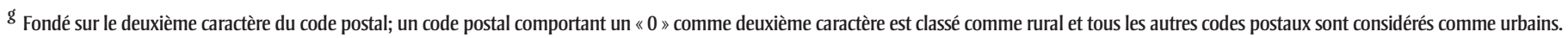

h Comprend six comtés : Hastings, Prince Edward, Lennox et Addington, Frontenac, Leeds-Grenville et Lanark.

en utilisant une procédure de sélection de variables ascendante et en précisant une valeur $p$ inférieure à 0,10 pour entrer et retenir les variables dans le modèle.

Toutes les références à la signification reposent sur des tests bilatéraux utilisant un coefficient alpha de 0,05.

\section{Résultats}

Le tableau 1 présente les distributions de fréquence de même que les âges moyens et médians au moment du diagnostic pour les variables indépendantes. Toutes les variables ont été retenues dans les modèles pour l'échantillon total et pour les sous-échantillons de la ColombieBritannique et du Manitoba, étant donné que les facteurs d'extension de la variance et les indices de condition étaient inférieurs aux valeurs indiquant des problèmes possibles de multicollinéarités1,32. Les tableaux 2 et 3 donnent les estimations de paramètres exponentielles tirées des modèles de régression, les résultats significatifs étant indiqués en caractères gras. Chaque estimation représente le ratio de l'âge attendu au moment du diagnostic à la catégorie de référence. Par exemple, l'estimation de paramètres exponentielle pour le syndrome d'Asperger est de 1,72 (tableau 2, modèles imputés). Cela signifie que les enfants atteints du syndrome d'Asperger ont, en moyenne, attendu 1,72 fois plus longtemps que ceux atteints d'un trouble autistique (la catégorie de référence) avant d’obtenir un diagnostic, lorsque toutes les autres variables demeuraient constantes.

Pour ce qui est de l'échantillon total, un diagnostic de TED-NS ou de syndrome d'Asperger, le fait d'habiter en milieu rural (modèles imputés), un diagnostic plus récent et le fait d'être né à l'étranger étaient associés de façon significative à un âge avancé au moment du diagnostic. À l'opposé, les enfants appartenant à une minorité visible ou ayant une sœur ou un frère atteint de TSA recevaient un diagnostic beaucoup plus tôt (tableau 2). La plupart des variables qui étaient significatives pour l'échantillon total le sont restées pour le sous-échantillon de la Colombie-Britannique, à l'exception de la résidence en milieu rural (tableau 3). Seuls le TED-NS, le syndrome d'Asperger et l'identité autochtone (modèles imputés) étaient significativement associés à l'âge au moment du diagnostic pour le sous-échantillon du Manitoba (tableau 3).
Lorsque les modèles étaient ajustés pour les sous-groupes de diagnostic, la seule variable qui demeurait significative était l'année du diagnostic. Les enfants faisant partie des deux derniers tiers de la période à l'étude avaient reçu un diagnostic beaucoup plus tard que ceux ayant obtenu un diagnostic entre 1997 et 1999 (données non présentées). Les autres variables qui ont montré des associations significatives dans l'analyse des sousgroupes de diagnostic étaient notamment les suivantes : le fait d'avoir été adopté, dans le cas du trouble autistique (estimation ponctuelle $=1,21$; intervalle de confiance [IC] à $95 \%: 1,01$ à 1,46$)$ et du TED-NS (estimation ponctuelle $=1,42$; IC à $95 \%$ : 1,11 à 1,83); le fait d'être né à l'étranger, dans le cas du trouble autistique (estimation ponctuelle $=1,45$; IC à $95 \%: 1,22$ à 1,72); et le fait d'avoir une sœur ou un frère atteint de TSA, dans le cas du TED-NS (estimation ponctuelle $=0,79$; IC à $95 \%$ : $0,67$ à 0,93$)$.

\section{Analyse}

Dans plusieurs études, le sous-groupe de diagnostic a été associé avec l'âge au moment du diagnostic de $\operatorname{TSA}^{13,17,33,34}$. 
TABLEAU 2

Ratio de l'âge attendu au moment du diagnostic de trouble du spectre autistique (TSA) par rapport à la catégorie de référence, pour l'échantillon total

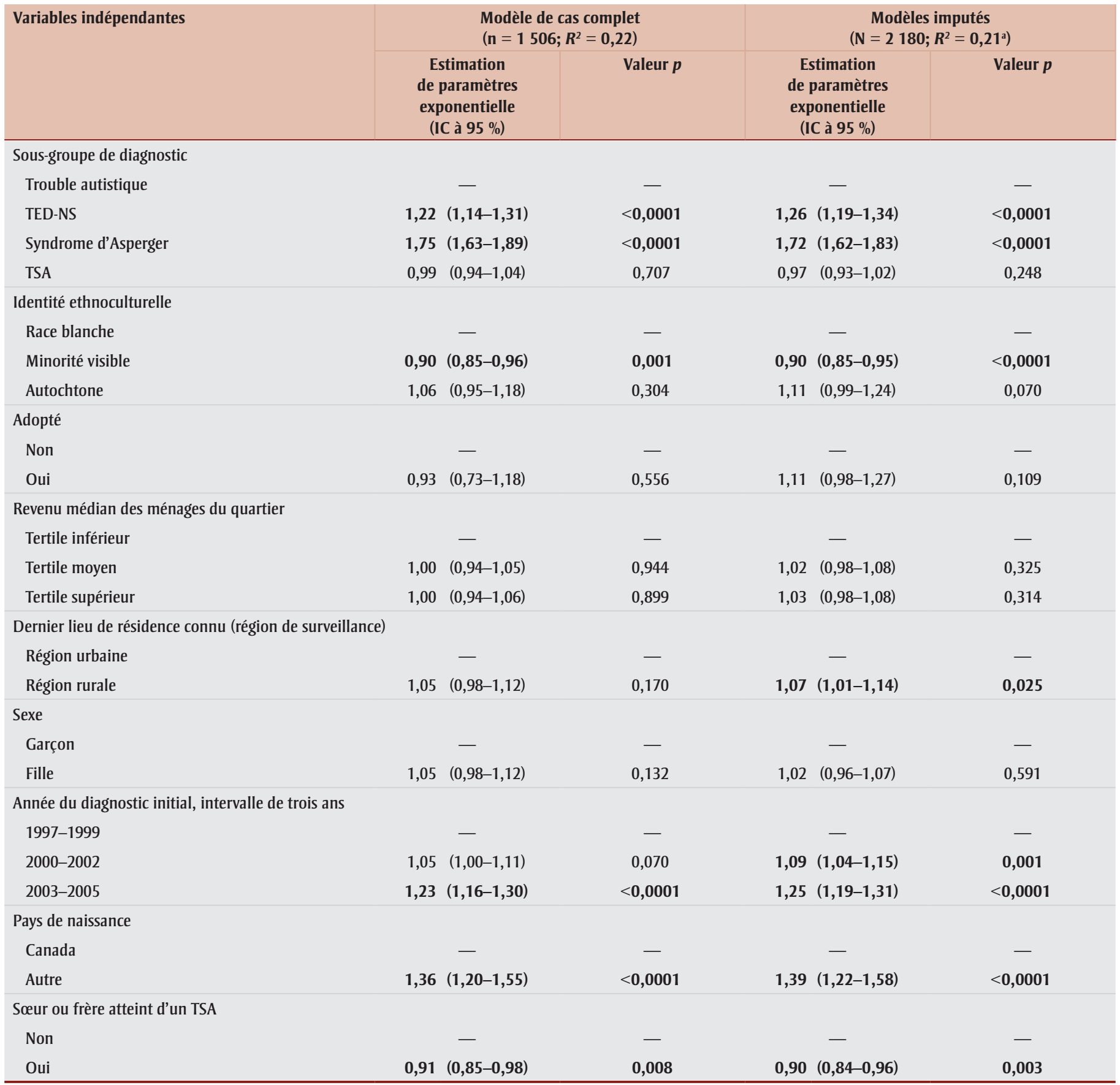

Abréviations : IC, intervalle de confiance; TED-NS, trouble envahissant du développement non spécifié; TSA, trouble du spectre autistique.

Remarque : Les catégories de référence sont indiquées par le tiret. Le caractère gras indique une association statistiquement significative selon $p<0,05$.

${ }^{\text {a }} R^{2}$ moyen pour 10 sous-ensembles avec des valeurs imputées pour les points de données manquants.

Dans notre échantillon également, les enfants atteints du syndrome d'Asperger ou d'un TED-NS recevaient généralement un diagnostic à un âge plus avancé que ceux atteints d'un trouble autistique (tableaux 2 et 3). Les variations de l'âge au moment du diagnostic observées entre les sous-groupes peuvent être attribuables aux différences de gravité des symptômes de base des TSA : en général, les enfants atteints d'un trouble autistique présentent davantage de retard sur le plan social, cognitif et de la communication que ceux atteints d'un TED-NS ou du syndrome
d'Asperger ${ }^{34}$, ce qui peut rapidement susciter de l'inquiétude chez les parents ou les professionnels.

Nous avons observé des associations significatives entre l'identité ethnoculturelle et l'âge au moment du diagnostic. On sait 
TABLEAU 3

Ratio de l'âge attendu au moment du diaǵnostic de trouble du spectre autistique (TSA) par rapport à la catégorie de référence, sous-échantillons de la Colombie-Britannique et du Manitoba

\begin{tabular}{|c|c|c|c|c|c|c|c|c|}
\hline & \multicolumn{4}{|c|}{ Colombie-Britannique } & \multicolumn{4}{|c|}{ Manitoba } \\
\hline & \multicolumn{2}{|c|}{$\begin{array}{l}\text { Modèle de cas complet } \\
\left.\text { (n=847; } R^{2}=0,15\right)\end{array}$} & \multicolumn{2}{|c|}{$\begin{array}{c}\text { Modèles imputés } \\
\left(\mathrm{n}=1247 ; R^{2}=0,15^{\mathrm{a}}\right)\end{array}$} & \multicolumn{2}{|c|}{$\begin{array}{l}\text { Modèle de cas complet } \\
\quad\left(\mathrm{n}=306 ; R^{2}=0,30\right)\end{array}$} & \multicolumn{2}{|c|}{$\begin{array}{c}\text { Modèles imputés } \\
\left(\mathrm{n}=493 ; R^{2}=0,35^{\mathrm{a}}\right)\end{array}$} \\
\hline & $\begin{array}{l}\text { Estimation de } \\
\text { paramètres } \\
\text { exponentielle } \\
\text { (IC à } 95 \% \text { ) }\end{array}$ & Valeur $p$ & $\begin{array}{l}\text { Estimation de } \\
\text { paramètres } \\
\text { exponentielle } \\
\text { (IC à } 95 \% \text { ) }\end{array}$ & Valeur $p$ & $\begin{array}{l}\text { Estimation de } \\
\text { paramètres } \\
\text { exponentielle } \\
\text { (IC à } 95 \%)\end{array}$ & Valeur $p$ & $\begin{array}{l}\text { Estimation de } \\
\text { paramètres } \\
\text { exponentielle } \\
\text { (IC à } 95 \%)\end{array}$ & Valeur $p$ \\
\hline \multicolumn{9}{|c|}{ Sous-groupe de diagnostic } \\
\hline Trouble autistique & - & - & - & - & - & - & - & - \\
\hline TED-NS & $1,14(1,03-1,26)$ & 0,012 & $1,16(1,07-1,26)$ & $<0,0001$ & $1,52(1,33-1,74)$ & $<0,0001$ & $1,62(1,44-1,83)$ & $<0,0001$ \\
\hline Syndrome d'Asperger & $1,62(1,45-1,81)$ & $<0,0001$ & $1,51 \quad(1,37-1,66)$ & $<0,0001$ & $1,86(1,62-2,14)$ & $<0,0001$ & $2,00 \quad(1,79-2,23)$ & $<0,0001$ \\
\hline TSA & $0,95 \quad(0,88-1,02)$ & 0,179 & $0,95 \quad(0,89-1,01)$ & 0,087 & $1,05 \quad(0,94-1,16)$ & 0,408 & $1,03 \quad(0,95-1,13)$ & 0,483 \\
\hline \multicolumn{9}{|l|}{ Identité ethnoculturelle } \\
\hline Race blanche & - & - & - & - & - & - & - & - \\
\hline Minorité visible & $0,87 \quad(0,82-0,94)$ & 0,0001 & $0,86(0,81-0,92)$ & $<0,0001$ & $0,96 \quad(0,86-1,09)$ & 0,554 & $0,93 \quad(0,83-1,05)$ & 0,258 \\
\hline Autochtone & $1,00 \quad(0,83-1,20)$ & 0,986 & $1,07 \quad(0,89-1,29)$ & 0,471 & $1,17 \quad(1,00-1,37)$ & 0,056 & $1,16(1,01-1,33)$ & 0,035 \\
\hline \multicolumn{9}{|l|}{ Adopté } \\
\hline Non & - & - & - & - & - & - & - & - \\
\hline Oui & $0,83 \quad(0,56-1,22)$ & 0,331 & $1,12 \quad(0,94-1,34)$ & 0,198 & $1,10 \quad(0,75-1,60)$ & 0,634 & $1,13 \quad(0,92-1,37)$ & 0,239 \\
\hline \multicolumn{9}{|c|}{ Revenu médian des ménages du quartier } \\
\hline Tertile inférieur & - & - & - & - & - & - & - & - \\
\hline Tertile moyen & $1,04 \quad(0,96-1,12)$ & 0,374 & $1,07 \quad(1,00-1,14)$ & 0,061 & $0,92(0,82-1,03)$ & 0,130 & $0,97 \quad(0,88-1,06)$ & 0,495 \\
\hline Tertile supérieur & $1,04 \quad(0,96-1,13)$ & 0,326 & $1,08(1,01-1,15)$ & 0,029 & $0,94 \quad(0,83-1,07)$ & 0,369 & $1,03 \quad(0,93-1,14)$ & 0,624 \\
\hline \multicolumn{9}{|c|}{ Dernier lieu de résidence connu (région de surveillance) } \\
\hline Région urbaine & - & - & - & - & - & - & - & - \\
\hline Région rurale & $1,02 \quad(0,89-1,18)$ & 0,731 & $1,07 \quad(0,96-1,19)$ & 0,208 & $1,08 \quad(0,97-1,21)$ & 0,177 & $1,09 \quad(0,99-1,20)$ & 0,093 \\
\hline \multicolumn{9}{|l|}{ Sexe } \\
\hline Garçon & - & - & - & - & - & - & - & - \\
\hline Fille & $1,00 \quad(0,92-1,10)$ & 0,939 & $0,97 \quad(0,90-1,04)$ & 0,390 & $1,08 \quad(0,94-1,23)$ & 0,273 & $1,05 \quad(0,94-1,17)$ & 0,374 \\
\hline \multicolumn{9}{|c|}{ Année du diagnostic initial, intervalle de trois ans } \\
\hline 1997-1999 & - & - & - & - & - & - & - & - \\
\hline $2000-2002$ & $1,05 \quad(0,97-1,14)$ & 0,203 & $1,12(1,05-1,21)$ & 0,001 & $0,98 \quad(0,87-1,10)$ & 0,740 & $1,03 \quad(0,92-1,15)$ & 0,654 \\
\hline 2003-2005 & $1,28 \quad(1,18-1,38)$ & $<0,0001$ & $1,33(1,24-1,42)$ & $<0,0001$ & $1,08 \quad(0,96-1,22)$ & 0,202 & $1,08 \quad(0,98-1,20)$ & 0,117 \\
\hline \multicolumn{9}{|l|}{ Pays de naissance } \\
\hline Canada & - & - & - & - & - & - & - & - \\
\hline Autre & $1,38(1,20-1,58)$ & $<0,0001$ & $1,38 \quad(1,22-1,57)$ & $<0,0001$ & $1,28 \quad(0,87-1,90)$ & 0,210 & $1,28 \quad(0,85-1,92)$ & 0,229 \\
\hline \multicolumn{9}{|c|}{ Sœur ou frère atteint d'un TSA } \\
\hline Non & - & - & - & - & - & - & - & - \\
\hline Oui & $0,89 \quad(0,80-1,00)$ & 0,052 & $0,83(0,75-0,92)$ & $<0,0001$ & $0,96 \quad(0,85-1,08)$ & 0,473 & $1,01 \quad(0,88-1,15)$ & 0,914 \\
\hline
\end{tabular}

Abréviations : IC, intervalle de confiance; TED-NS, trouble envahissant du développement non spécifié; TSA, trouble du spectre autistique.

Remarque : Les catégories de référence sont indiquées par le tiret. Le caractère gras indique une association statistiquement significative selon $p<0,05$.

${ }^{\text {a }} R^{2}$ moyen pour 10 sous-ensembles avec des valeurs imputées pour les points de données manquants.

peu de chose sur l'influence des facteurs culturels sur le moment où les enfants atteints de TSA reçoivent un diagnostic. Selon une étude néerlandaise récente, les minorités ethniques sont sous-représentées sur le plan de l'aiguillage vers des centres d'évaluation des $\mathrm{TSA}^{35}$. Les auteurs ont fait remarquer que les pédiatres attribuaient peut-être les retards de ces enfants sur le plan social et de la communication à des facteurs culturels ${ }^{35}$. Une telle explication n'est pas conforme à nos constatations, selon lesquelles le diagnostic est posé plus tôt chez les minorités visibles dans l'échantillon total (tableau 2) et dans le sous-échantillon des cas de la ColombieBritannique (tableau 3); toutefois, cela pourrait expliquer en partie le fait qu'au 
Manitoba, les enfants autochtones faisaient souvent l'objet d'un diagnostic plus tardif que les enfants de race blanche (tableau 3). Mandell et collab. ont constaté que les effets de l'ethnicité sur l'âge au moment du diagnostic étaient plus prononcés chez les enfants atteints de troubles du spectre autistique au sens large (c.-à-d. ceux chez qui on a posé un diagnostic de syndrome d'Asperger ou de TED-NS) ${ }^{19}$. Nous n'avons pas inclus de termes d'interaction dans les modèles de régression multiple, mais nous n'avons observé aucun effet significatif de l'identité ethnoculturelle sur l'âge au moment du diagnostic lorsque nous avons effectué une stratification selon le sous-groupe de diagnostic (données non présentées). Par conséquent, l'influence de l'identité ethnoculturelle sur l'âge, dans notre échantillon, ne semblait pas concentrée à une extrémité du spectre autistique.

On croit également que les symptômes de TSA chez les enfants adoptés pourraient être attribués à tort aux expériences vécues pendant la petite enfance, ce qui aurait pour effet de retarder le diagnostic ${ }^{17}$. Même si nous n'avons observé aucune association entre l'adoption et l'âge au moment du diagnostic dans l'échantillon total ni dans les souséchantillons de la Colombie-Britannique et du Manitoba, cette association était significative pour les sous-échantillons du trouble autistique et du TED-NS. Par conséquent, il faudrait envisager d'inclure cette variable dans les études futures qui porteront sur l'examen des facteurs liés au moment du diagnostic.

Contrairement à plusieurs études américaines $^{17,21}$, nous n'avons observé aucune association entre le revenu et l'âge au moment du diagnostic dans notre échantillon (sauf dans les modèles imputés pour la Colombie-Britannique, où l'association allait dans le sens contraire de ce qui était attendu : les enfants vivant dans des quartiers affichant les revenus médians les plus élevés recevaient généralement un diagnostic plus tardif que ceux des quartiers dont les revenus médians étaient les plus faibles). La différence entre nos observations et celles des études américaines peut s'expliquer de deux façons. Premièrement, les études américaines ont utilisé des mesures du revenu individuel, tandis que nous avons utilisé une mesure du revenu à l'échelle du quartier comme variable de substitution du revenu des ménages. Deuxièmement, il est possible que nous n’ayons observé aucune association parce que l'accès aux services de santé est généralement plus facile sur le plan financier au Canada qu'aux États-Unis.

Mandell et collab. ont également signalé que les enfants des régions rurales recevaient souvent un diagnostic de TSA plus tardivement que les enfants vivant dans des régions urbaines $^{17}$. Selon leur hypothèse, une plus forte densité de population contribue à créer une masse critique d'enfants atteints de TSA, ce qui mène à une meilleure connaissance du trouble de la part des professionnels de la santé et des familles, d'où la reconnaissance rapide du problème. En outre, il peut être plus difficile pour les enfants des régions rurales d'avoir accès à des services de diagnostic spécialisés. Si ce facteur constitue un déterminant majeur des retards de diagnostic, on pourrait s'attendre à observer un lien entre le fait d'habiter en milieu rural et l'âge au moment du diagnostic au Manitoba : la superficie du territoire couvre presque $650000 \mathrm{~km}^{2}$, et les deux principaux centres de référence et d'évaluation pour les enfants que l'on soupçonne d'être atteints de TSA sont situés à Winnipeg. Toutefois, même si les enfants des régions rurales étaient plus susceptibles de recevoir un diagnostic tardif que ceux des régions urbaines dans tous les modèles, l'association n'était significative que dans les modèles imputés pour l'échantillon total. Il faudrait, dans des études ultérieures, prendre en compte la distance pour se rendre aux centres de référence et d'évaluation spécialisés plutôt que la résidence en milieu urbain par rapport à la résidence en milieu rural, chose que nous n'avons pu faire car notre base de données ne contient aucune adresse.

En raison du plus grand nombre de filles que de garçons composant notre échantillon ( $n=371$ ) et de l'absence d'association entre le sexe et l'âge au moment du diagnostic dans l'ensemble des analyses, rien n'indiquait la présence de ce que Shattuck et collab. ont appelé un [traduction] «biais lié au sexe dans les attentes culturelles à l'égard du comportement des enfants ou dans les pratiques cliniques en matière de dépistage, d'aiguillage et de diagnostic $»^{16}$. Il est moins encourageant de constater que l'âge au moment du diagnostic a augmenté au cours de la période à l'étude dans l'échantillon total, même si les données de la Colombie-Britannique, région ayant la plus grande taille d'échantillon, pourraient être responsable au premier chef de cette tendance; aucune augmentation significative n’a été constatée au Manitoba (tableau 3). (Une augmentation temporelle de l'âge au moment du diagnostic de TSA a également été signalée pour le sud-est de l'Ontario ${ }^{14}$.) Le diagnostic a été posé plus tardivement dans une proportion d'environ $25 \%$ pour la période 2003-2005 comparativement à 1997-1999 (tableau 2). On a observé un profil semblable dans tous les sous-groupes de diagnostic (données non présentées); par conséquent, il est peu probable qu'on puisse attribuer ce résultat uniquement à une hausse de l'aiguillage des enfants plus âgés présentant des symptômes plus légers dans les dernières années de la période à l'étude. Il est possible que les services d'évaluation offerts dans certaines régions soient devenus engorgés en raison du nombre accru d'aiguillages au cours de la période à l'étude, entraînant ainsi des périodes d'attente plus longues. Même si nous n'avons pas les données pour évaluer cette hypothèse, le Comité permanent des affaires sociales, des sciences et de la technologie du Sénat a noté, dans son rapport paru en 2007 de l'enquête portant sur le financement du traitement des TSA au Canada, que les parents d'enfants atteints de TSA avaient souvent de la difficulté à obtenir des services de diagnostic et d'évaluation dans des délais rapides ${ }^{36}$.

Nous avons examiné deux facteurs - le lieu de naissance et le fait d'avoir une sœur ou un frère atteint de TSA - qui n'avaient pas, à notre connaissance, été inclus dans d'autres études de l'âge au moment du diagnostic de TSA. Les enfants nés à l'étranger étaient plus nombreux à faire l'objet d'un diagnostic tardif que ceux nés au Canada. Cette variable comportait l'un des plus grands effets, venant tout juste derrière un diagnostic de syndrome d'Asperger (tableau 2). Il serait intéressant d'étudier cette association dans un plus grand échantillon comportant plus de catégories discrètes pour le lieu de naissance. Les résultats pourraient servir de base à la formulation de recommandations visant à améliorer les services de dépistage et 
d'évaluation parmi les groupes pouvant être sujets à un dépistage tardif, de manière à ce que tous les enfants atteints de TSA reçoivent un diagnostic le plus rapidement possible.

Le fait d'avoir une sœur ou un frère atteint de TSA était associé à un âge précoce au moment du diagnostic dans l'échantillon total et en Colombie-Britannique (tableaux 2 et 3), même si cette variable n'était significative que pour le sous-groupe de TED-NS. Cette observation laisse croire que les symptômes plus légers de TED-NS susciteraient plus rapidement de l'inquiétude losrque l'enfant a un frère ou une sœur ayant déjà reçu un diagnostic de TSA, alors que les symptômes d'un trouble autistique, en revanche, devraient être assez graves pour que les parents ou les professionnels s'en inquiètent rapidement, qu'il y ait ou non un autre enfant atteint d'un TSA dans la famille. Si cette hypothèse était juste, on s'attendrait à ce que cette variable soit également significative pour le syndrome d'Asperger, or ce n'est pas le cas, constat qui pourrait être attribuable au petit nombre d'enfants dans ce groupe qui ont une sœur ou un frère atteint de TSA $(n=16)$. Pour mieux évaluer cette hypothèse, il serait utile d'inclure dans de prochaines études des renseignements permettant de savoir si les frères et sœurs sont plus vieux ou plus jeunes que l'enfant (nous n'avons pas recueilli ces renseignements dans la NEDSAC avant 2009).

Un de nos objectifs était de déterminer si les facteurs examinés pour l'échantillon total avaient un effet différentiel sur l'âge au moment du diagnostic à l'échelle régionale. Les seules associations significatives communes à la Colombie-Britannique et au Manitoba concernaient le sous-groupe de diagnostic (tableau 3). Cette observation souligne la nécessité de tenir compte de la façon dont l'agrégation des données peut masquer les différences entre les régions. Si les résultats provenant d'autres analyses semblables visent à orienter les politiques et la pratique clinique à l'échelle locale, les stratégies analytiques devraient être utilisées avec cet objectif en tête. Cet aspect est particulièrement pertinent au Canada, où les services de diagnostic varient énormément d'une province à l'autre. En 2003, par exemple, le gouvernement de la Colombie-Britannique a mis sur pied un réseau provincial de cliniciens qui utilisent des lignes directrices normalisées pour évaluer les enfants et les adolescents que l'on soupçonne de présenter des TSA et pour poser un diagnostic. À l'opposé, les principaux centres de référence et d'évaluation du Manitoba sont situés à Winnipeg. Il n'est donc pas étonnant que les facteurs qui influent sur le moment où un enfant atteint de TSA reçoit un diagnostic soient différents d'une province à l'autre.

Les valeurs de $R^{2}$ indiquent que les variables examinées on à peine compté dans la variation de l'âge au moment du diagnostic (15\% à $35 \%$; tableaux 2 et 3 ). À certains égards, ce constat est rassurant : les caractéristiques sociodémographiques et socio-économiques n'exerceraient pas une grande influence sur le moment où l'enfant reçoit un diagnostic de TSA au Canada. En revanche, cela signifie qu'il reste beaucoup à faire pour déterminer les facteurs qui contribuent aux différences observées dans l'âge au moment du diagnostic. Mandell et collab. ont procédé à une régression d'un grand nombre de variables individuelles sur l'âge au moment du diagnostic, notamment les symptômes des TSA, la présence chez l'enfant d'une déficience intellectuelle, d'une déficience auditive ou d'une maladie épileptique, le nombre de médecins consultés avant le diagnostic, la réalisation ou non de tests d'évaluation du développement et l'aiguillage ou non vers un spécialiste ${ }^{17}$. Dans leur modèle, $46 \%$ de la variation de l'âge au moment du diagnostic est demeurée inexpliquée. Au moins deux études ont examiné l'influence des facteurs régionaux sur l'âge au diagnostic ${ }^{19,21}$. Dans ces deux cas, la plus grande partie de la variation de l'âge au moment du diagnostic était associée aux différences individuelles. Toutefois, les facteurs régionaux examinés étaient essentiellement de nature socio-économique et sociodémographique. Nous aimerions, lors de futures études, déterminer si les caractéristiques du système de santé sont des déterminants majeurs du moment où les enfants reçoivent un diagnostic de TSA. Ces caractéristiques pourraient comprendre le nombre de pédiatres spécialistes du développement par habitant, l'âge moyen des pédiatres qui exercent dans la région, l'existence ou non de paramètres de pratique en place pour le dépistage des TSA et le temps moyen d'attente pour obtenir une évaluation.

\section{Points forts et limites}

Une des limites de la présente étude est l'absence de données cliniques sur l'état cognitif, les affections concomitantes et les symptômes des TSA, facteurs qui, dans plusieurs études américaines, étaient fortement associés avec l'âge au moment du diagnostic ${ }^{16,17}$. En outre, même si nos protocoles de surveillance étaient conçus de manière à obtenir des données sur la plupart des enfants chez qui un TSA avait été diagnostiqué au Manitoba, dans le sud-est de l'Ontario, à l'Île-du-Prince-Édouard et à Terre-Neuve-et-Labrador, les données de la Colombie-Britannique et de Calgary proviennent principalement des cliniques. Par conséquent, nous ne connaissons pas la portée de notre détermination des cas dans ces régions. Une autre limite possible concerne la méthode d'imputation multiple dans le système SAS, qui suppose que certaines valeurs sont manquantes de façon aléatoire. Il n'est pas possible habituellement de le vérifier, mais les hypothèses erronées à cet égard pourraient avoir une incidence mineure sur les estimations des paramètres et les écarts-types ${ }^{37}$. L'algorithme de Monte Carlo par chaîne de Markov suppose une distribution normale à plusieurs variables pour imputer les valeurs manquantes. Toutefois, il a été démontré qu'il fonctionne généralement pour les variables nominales si les valeurs imputées ne sont pas arrondies $^{29}$. C'est la méthode que nous avons utilisée.

Comme le montre la figure 1, nous avons exclu les cas pour lesquels le mois et l'année du diagnostic initial étaient inconnus (ce qui nous aurait empêchés de calculer l'âge au moment du diagnostic) et les cas pour lesquels il était impossible de confirmer la résidence dans les régions de surveillance durant l'année du diagnostic initial. Nous ignorons quelle proportion de ces cas aurait satisfait aux critères d'inclusion dans l'échantillon; toutefois, rien ne permet de croire que les enfants qui ont reçu un diagnostic de 1997 à 2005 et qui n'étaient pas inclus dans cette analyse pour l'un des motifs précédents étaient 
systématiquement différents de l'échantillon en ce qui a trait aux variables indépendantes et à l'âge où ils avaient reçu un diagnostic de TSA. Néanmoins, nous ne pouvons écarter la possibilité d'un biais dans nos estimations. Par conséquent, ce travail doit être reproduit dans d'autres échantillons, nos résultats pouvant aider à orienter de telles recherches.

Parmi les forces de la présente étude, mentionnons la grande taille de l'échantillon, qui a permis d'obtenir une bonne puissance statistique pour examiner l'association entre un certain nombre de facteurs et l'âge auquel les enfants ont reçu un diagnostic de TSA. À notre connaissance, notre étude est la première à examiner le lieu de naissance à l'étranger (de l'enfant) et la présence d'une sœur ou d'un frère atteint de TSA par rapport à l'âge au moment du diagnostic, et il s'agit de la première analyse du genre dans la population canadienne. Les grandes tailles d'échantillon pour la Colombie-Britannique et le Manitoba nous ont permis d'effectuer des analyses distinctes pour ces deux régions, qui ont révélé certaines différences qui pourraient être importantes quant à la façon dont certains facteurs sont liés à l'âge au moment du diagnostic à l'échelle régionale. Une autre force de notre analyse est le fait que nous avons inclus uniquement les enfants qui résidaient dans les régions faisant l'objet d'une surveillance au moment du diagnostic, ce qui augmente la probabilité que les résultats correspondent à la situation de la région en matière de diagnostic durant la période à l'étude.

L'une des constatations les plus intrigantes est peut-être la faible proportion de la variation de l'âge au moment du diagnostic qui était expliquée par nos modèles. Même si cette observation laisse entendre que les facteurs socio-économiques et sociodémographiques examinés ont eu peu d'impact sur le moment où les enfants atteints de TSA ont reçu un diagnostic - alors que, d'après une étude américaine récente, ces facteurs étaient généralement des prédicteurs plus solides de l'âge au moment du diagnostic que la gravité des symptômes ${ }^{21}$ elle souligne bien la nécessité de mieux comprendre les facteurs qui contribuent aux disparités relatives à l'âge au moment du diagnostic. Les prochaines études devraient inclure des renseignements plus détaillés sur les variables que nous avons examinées, de même qu'un éventail plus large de facteurs. Ces facteurs pourraient comprendre des caractéristiques individuelles, comme la présence d'affections cliniques concomitantes qui pourraient vraisemblablement retarder la reconnaissance des symptômes comportementaux liés aux $\mathrm{TSA}^{38}$, ainsi que les caractéristiques du système de santé, comme le temps d'attente pour être aiguillé et évalué. De telles études sont cruciales pour assurer un accès rapide au traitement et à des services de soutien pour tous les enfants atteints de TSA et leurs familles.

\section{Remerciements}

Nous tenons à remercier les familles et les organismes de chaque région qui ont fourni des renseignements à la National Epidemiologic Database for the Study of Autism in Canada (NEDSAC). Il est possible de consulter la liste des organismes et des partenaires à l'adresse www.nedsac.ca. Nous souhaitons également remercier Services spéciaux pour enfants et Services à la famille et Consommation du Manitoba pour leur aide à la collecte des données; Robert Gauthier et Lori Crews, anciens codirecteurs régionaux de la NEDSAC à Terre-Neuve-et-Labrador; l'Autism Calgary Association; la Society for Treatment of Autism; les cliniques du développement de l'Alberta Children's Hospital; le $\mathrm{D}^{\mathrm{r}}$ Jean François Lemay, département de pédiatrie, Université de Calgary et Alberta Children's Hospital, ainsi que les membres du British Columbia Autism Spectrum Interdisciplinary Research (ASPIRE) Program (Directeur : MES Lewis; appuyé financièrement par une bourse de la Fondation Michael Smith pour la recherche en santé) pour leur soutien et leur aide. Les résultats et les interprétations présentés dans la présente étude sont ceux des auteurs et ne reflètent pas nécessairement les opinions des personnes ni celles des organismes qui ont fourni les données nécessaires à ce projet.

La présente étude a bénéficié d'une subvention d'équipe interdisciplinaire de recherche en santé financée par les Instituts de recherche en santé du Canada ( $n^{\circ}$ 43820), octroyée au Autism Spectrum Disorders-Canadian-American
Research Consortium (ASD-CARC : www.asdcarc.com; J. J. Holden, chercheur principal), et d'une subvention d'exploitation des Instituts de recherche en santé du Canada (n 79556) octroyée à H. Ouellette-Kuntz.

\section{Références}

1. Autism and Developmental Disabilities Monitoring Network Surveillance Year 2002 Principal Investigators. Centers for Disease Control and Prevention. Prevalence of autism spectrum disorders-Autism and Developmental Disabilities Monitoring Network, 14 sites, United States, 2002. MMWR. 2007;56(SS01):12-28.

2. Johnson CP, Myers SM, American Academy of Pediatrics Council on Children With Disabilities. Identification and evaluation of children with autism spectrum disorders. Pediatrics 2007;20(5):1183-215.

3. Instituts de recherche en santé du Canada. La recherche en santé : la clé pour améliorer le sort des enfants autistes [Internet]. Ottawa (Ontario) : Instituts de recherche en santé du Canada, 2006 [consultation le 8 fév. 2010]. Consultable en ligne à la page : http://www.scoop-sante.com /communique-27448.html

4. Barbaro J, Dissanayake C. Autism spectrum disorders in infancy and toddlerhood: a review of the evidence on early signs, early identification tools, and early diagnosis. J Dev Behav Pediatr. 2009;30(5):447-59.

5. Eldevik S, Hastings RP, Hughes JC, Jahr E, Eikeseth S, Cross S. Meta-analysis of Early Intensive Behavioral Intervention for children with autism. J Clin Child Adolesc Psychol. 2009;38(3):439-50.

6. Dawson G. Early behavioral intervention, brain plasticity, and the prevention of autism spectrum disorder. Dev Psychopathol. 2008;20(3):775-803.

7. Fenske EC, Zalenski S, Krantz PJ, McClannahan LE. Age at intervention and treatment outcome for autistic children in a comprehensive intervention program. Anal Intervention Dev Dis. 1985;5(1-2):49-58.

8. Harris SL, Handleman JS. Age and IQ at intake as predictors of placement for young children with autism: a four- to six-year follow up. J Autism Dev Disord. 2000;30(2):137-42. 
9. British Columbia Ministry of Children and Family Development. Fact sheet: Autism spectrum disorder [Internet]. Victoria (BC): British Columbia Ministry of Children and Family Development; 2010 [consultation le 24 juint 2011]. PDF (142 Ko) téléchargeable à partir du lien : http: //www.mcf.gov.bc.ca/spec_needs/pdf /Factsheet_Autism_Jan \%202010_FNL.pdf

10. Midence K, O’Neill M. The experience of parents in the diagnosis of autism: a pilot study. Autism. 1999;3(3):273-85.

11. Cox A, Klein K, Charman T, Baird G, Baron-Cohen S, Swettenham J et collab. Autism spectrum disorders at 20 and 42 months of age: stability of clinical and ADI-R diagnosis. J Child Psychol Psychiatry. 1999;40(5):719-32.

12. Moore V, Goodson S. How well does early diagnosis of autism stand the test of time? Follow-up study of children assessed for autism at age 2 and development of an early diagnostic service. Autism. 2003;7(1):47-63.

13. Wiggins LD, Baio J, Rice C. Examination of the time between first evaluation and first autism spectrum diagnosis in a population-based sample. J Dev Behav Pediatr. 2006;27(2 Suppl):S79-S87.

14. Ouellette-Kuntz HM, Coo H, Lam M, Yu CT, Breitenbach MM, Hennessey PE et collab. Age at diagnosis of autism spectrum disorders in four regions of Canada. Can J Public Health. 2009;100(4):268-73.

15. Offord Centre for Child Studies. The sooner you know, the sooner you can help: CAIRN launches awareness campaign to identify autism earlier [Internet]. Hamilton (Ont.): Offord Centre for Child Studies; 2004 [consultation le 10 janv. 2008]. Consultable à la page : http://www.offordcentre.com /asd/asd_art3.html

16. Shattuck PT, Durkin M, Maenner M, Newschaffer C, Mandell DS, Wiggins L et collab. Timing of identification among children with an autism spectrum disorder: findings from a population-based surveillance study. J Am Acad Child Adolesc Psychiatry. 2009;48(5):474-83.

17. Mandell DS, Novak MM, Zubritsky CD. Factors associated with age of diagnosis among children with autism spectrum disorders. Pediatrics. 2005;116(6):1480-6.
18. Rhoades RA, Scarpa A, Salley B. The importance of physician knowledge of autism spectrum disorder: results of a parent survey. BMC Pediatr. 2007;7(37).

19. Mandell DS, Morales KH, Xie M, Lawer LJ, Stahmer AC, Marcus SC. Age of diagnosis among Medicaid-enrolled children with autism, 2001-2004. Psychiatr Serv. 2010;61(8):822-9.

20. Giarelli E, Wiggins LD, Rice CE, Levy SE, Kirby RS, Pinto-Martin J et collab. Sex differences in the evaluation and diagnosis of autism spectrum disorders among children. Disabil Health J. 2010;3(2):107-16.

21. Fountain C, King MD, Bearman PS. Age of diagnosis for autism: individual and community factors across 10 birth cohorts. J Epidemiol Community Health. 2011;65(6):503-10.

22. Mandell D, Listerud J, Levy SE, Pinto-Martin JA. Race differences in the age at diagnosis among Medicaid-eligible children with autism. J Am Acad Child Adolesc Psychiatry. 2002; 41(12):1447-53.

23. Ouellette-Kuntz H, Coo H, Yu CT, Lewis ME, Dewey D, Hennessey PE et collab. National Epidemiologic Database for the Study of Autism in Canada (NEDSAC). Maladies chroniques et blessures au Canada. 2012;32(2):95-101.

24. Statistique Canada. Profil : revenu des particuliers, des familles et des ménages, caractéristiques socioéconomiques des particuliers, des familles et des ménages, coûts d'habitation, et religion, pour le Canada, les provinces, les territoires et les régions de tri d'acheminement, Recensement de 2001 [base de données]. Ottawa (Ont.) : Statistique Canada; publié le 17 juillet 2003 (série « Profils » : 95f0492xcb01003).

25. Statistique Canada. Mise en correspondance des codes postaux et des régions géographiques. Glossaire [Internet]. Ottawa (Ont.) : Statistique Canada; 2007 [consultationle 25 oct. 2007]. PDF (316 Ko) téléchargeable à partir du lien : http://www.statcan.gc.ca /pub/92f0138m/92f0138m2007001-fra.pdf

26. Manning WG, Mullahy J. Estimating log models: to transform or not to transform? J Health Econ. 2001;20(4):461-94.
27. Afifi AA, Kotlerman JB, Ettner SL, Cowan M. Methods for improving regression analysis for skewed continuous or counted responses. Annu Rev Public Health. 2007;28:95-111.

28. SAS Institute. Multiple imputation for missing data [Internet]. Cary (NC): SAS Institute; 2009 [consultationle 9 mars 2009]. Consultable à la page : http://support.sas.com /rnd/app/da/new/dami.html

29. Allison PD. Paper 113-30. Imputation of categorical variables with PROC MI. Paper presented at: Proceedings of the Thirtieth Annual SAS Users Group International Conference; 2005 Apr 10; Cary (NC).

30. Zheng B. Summarizing the goodness of fit of generalized linear models for longitudinal data. Stat Med. 2000;19(10):1265-75.

31. Katz MH. Multivariable Analysis. A Practical Guide for Clinicians. Cambridge (UK): Cambridge University Press; 2006.

32. SAS Institute. SAS/STAT(R) 9.2 User's Guide, Second Edition [Internet]. Cary (NC): SAS Institute; 2009 [consultationle 29 mars 2010]. Consultable à la page : http://support.sas.com /documentation/cdl/en/statug/63033 /HTML/default/viewer.htm\#/documentation /cdl/en/statug/63033/HTML/default /statug_reg_sect038.htm

33. Howlin P, Asgharian A. The diagnosis of autism and Asperger syndrome: findings from a survey of 770 families. Dev Med Child Neurol. 1999;41(12):834-9.

34. Chakrabarti S, Fombonne E. Pervasive developmental disorders in preschool children: confirmation of high prevalence. Am J Psychiatry. 2005;162(6):1133-41.

35. Begeer S, Bouk SE, Boussaid W, Terwogt MM, Koot HM. Underdiagnosis and referral bias of autism in ethnic minorities. J Autism Dev Disord. 2009;39(1):142-8.

36. Eggleton A, Keon WJ. Rapport final sur l'interpellation au sujet du financement pour le traitement de l'autisme - Payer maintenant ou payer plus tard : les familles d'enfants en crise [Internet]. Ottawa (Ont.) : Le Comité sénatorial permanent des affaires sociales, des sciences et de la technologie, 2007 [consultationle 6 novembre 2007]. Consultable à la page http://www.parl.gc.ca /Content/SEN/Committee/391/soci/rep /repfinmar07-f.htm 
37. Schafer JL, Graham JW. Missing data: our view of state of the art. Psychol Methods. 2002;7(2):147-77.

38. Schaefer GB, Mendelsohn NJ. Professional Practice and Guidelines Committee. Clinical genetics evaluation in identifying the etiology of autism spectrum disorders. Genet Med. 2008;10(4):301-5. 